\title{
Erkranken Kinder an der deutschen Umwelt?
}

D hat as Berliner Robert-Koch-Institut hat am 12. März die erste Phase der „Studie zur Gesundheit von Kindern und Jugendlichen in Deutschland“ eingeleitet. Die Untersuchung soll zeitliche und regionale Entwicklungen, Häufigkeit von Krankheiten sowie Schadstoffbelastungen aufzeigen. Ziel ist, die Grundlagen für gesundheitspolitische Entscheidungen zu verbessern.

Die Studie soll insgesamt fünf Jahre dauern. Die erste Phase, in der vor allem Berliner Haushalte befragt werden, endet im Frühjahr 2002. Ab August 2002 startet die Hauptphase, in der bundesweit ca. 20.000 Kinder bis 18 Jahre und ihre Eltern in 150 Orten Auskunft geben sollen. Die Hauptphase dauert drei Jahre.

Die Ministerien für Gesundheit sowie für Bildung und Forschung finanzieren gemeinsam die Studie. Das Bundesministerium für Umwelt, Naturschutz und Reaktorsicherheit unterstützt eine Zusatzerhebung mit Schadstoffuntersuchungen und Lärmmessungen im häuslichen Umfeld.

Nach Informationen der „Partner für

Berlin Gesellschaft".

\section{Rauchen \& Asthma er- höht Frühgeburt-Risiko}

\begin{abstract}
rühgeburt und andere perinatale Komplikationen sind bei rauchenden Müttern mit Asthma doppelt so häufig wie bei nicht rauchenden Asthma-Patientinnen. Das Ergebnis der retrospektiven Studie stellte Kristin Kloos von der Mayo Clinic auf dem diesjährigen AAAAI-Kongress in New Orleans vor. Bei der Analyse von 702 Krankenakten zeigte sich zudem, dass $13 \%$ der Frauen ohne Asthma rauchten. Im Vergleich dazu war fast jede fünfte Frau mit Therapie-pflichtigem Asthma Raucherin.
\end{abstract}

\section{Kloos et al.}

AAAAI-Kongress 2001, New Orleans.

\section{Tee antiallergisch bei atopischem Ekzem}

aut einer japanischen Studie löscht - Tee nicht nur den Durst, sondern auch den Juckreiz bei atopischem Ekzem (AE). Wissenschaftler der Universitäts-Hautklinik in Otsu verschrieben Tee für 118 Patienten mit Therapierefraktärem $\mathrm{AE}$, die sie mindestens ein halbes Jahr lang behandelt hatten (topische Steroide, orale Antihistaminika und Allergen-Karenz). Die Probanden tranken drei Mal täglich eine definierte Dosis Oolong-Tee. Die Wissenschaftler fotografierten zwei bis drei repräsentative Läsionen zu Studienbeginn, nach vier sowie nach 24 Wochen. Auf einer Lickert-Skala dokumentierten die Patienten die Veränderung des Juckreizes.

Bereits nach zwei Wochen beobachteten die Dermatologen einen posi-
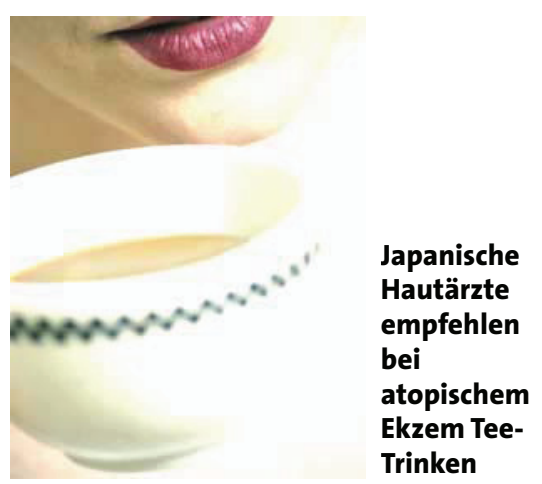

tiven Effekt ihrer Tee-Kur. Nach einem Monat zeigten 63\% der Patienten eine mäßige bis deutliche Verbesserung. Nach sechs Monaten waren es noch 54\%. Nur schade, dass die Studie die Kriterien der „Evidence Based Medicine" nicht erfüllt.

Uehara M et al. Arch Dermatol 2001; 137: 42-3.

\section{Allergen-Reduktion lohnt bei Risiko-Kindern}

Britische Wissenschaftler bestätigen, dass sich Allergien und Asthma bei familiär belasteten Kindern durch konsequente Primärprävention vermeiden lassen. Arshad et al. verglichen Risiko-Kinder mit geringer bzw. normaler Allergenbelastung. In der ersten Gruppe stillten die Mütter und ernährten sich hypoallergen oder sie gaben dem Kind stark hydrolysierte Säuglingsnahrung. Außer- dem achteten sie darauf, Hausstaubmilben vorzubeugen. Die Kontrollgruppe befolgte keine besonderen Präventionsmaßnahmen. Bei Einjährigen der ersten Gruppe traten Asthma-assoziierte Symptome signifikant seltener auf als bei den Kindern der Kontrollgruppe.

\section{Arshad et al.}

AAAAI-Kongress 2001, New Orleans.

\section{Schallwellen erkennen ASS-Intoleranz}

$\mathrm{D}$ ie akustische Rhinometrie ist hinreichend genau zur Diagnose einer ASS-Intoleranz. Sie wird selbst von Patienten mit starker bronchialer Obstruktion gut vertragen. Das ist das Ergebnis einer Studie an 15 Patienten mit Asthma und Rhinitis aufgrund einer ASS-Unverträglichkeit, acht $\mathrm{Pa}$ tienten mit den gleichen Symptomen, aber ohne Intoleranz, sowie acht gesunden Probanden. Die Studienteilnehmer sprühten an zwei aufeinander folgenden Tagen 25 mg Lysin-AzetylsalizylsäureLösung in jedes Nasenloch.
Bei den Patienten mit ASS-Intoleranz rief die Provokation eine signifikante Zunahme von Fließschnupfen, Niesen und Obstruktion hervor. Die übrigen Probanden zeigten keine Reaktion. Die akustische Rhinometrie, die durch Schallreflexion die nasale Geometrie misst, ergab bei den betroffenen Patienten eine Verminderung des Nasalvolumens. Die Methode sei mit einer Spezifität von $94 \%$ der Rhinomanometrie überlegen.

\section{tp}

Casadevall J et al. Thorax 2000; 55: 921-4. 